PAPER

\title{
Heralding manifestations of basilar artery occlusion with lethal or severe stroke
}

\author{
G von Campe, F Regli, J Bogousslavsky
}

J Neurol Neurosurg Psychiatry 2003;74:1621-1626

See end of article for authors' affiliations

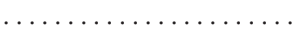

Correspondence to:

Professor Julien

Bogousslavsky, Service de Neurologie, CHUV BH-13, $\mathrm{CH}-1011$ Lausanne,

Switzerland

Received 29 May 2003

Accepted 17 June 2003

\begin{abstract}
Background: Basilar artery occlusion usually causes severe disability or death. Until the recent developments in local intra-arterial or systemic intravenous fibrinolysis, interest in early diagnosis was low because there was no satisfactory treatment. Thus there is little information about the initial phase of the disease.

Objective: To report on the early clinical features and patterns of evolution of severe symptomatic basilar artery occlusion.

Methods: 24 patients with established basilar artery occlusion (confirmed by angiography or at necropsy) were reviewed retrospectively, focusing on the early clinical aspects and time course of the disease.

Results: The most common initial symptoms were motor deficits (16/24, including facial palsies), articulatory speech difficulties (15/24), vertigo, nausea or vomiting (13/24), and headaches (10/24). The most frequent objective initial findings were motor deficits (22/24), facial palsies (19/24), eye movement abnormalities (15/24), lower cranial nerve deficits (15/24), altered level of consciousness (12/24), and bilateral extensor plantar responses (9/24). Onset of the disease was gradual in nearly all patients and in half the warning signs were present for up to two months before the final stage. Headaches and visual disturbances were early signs, while speech difficulties and motor deficits were late signs. Once permanent neurological deficits were present, the final illness was reached within six hours in $41 \%$, between six and 24 hours in $32 \%$, and in two to three days in $27 \%$.

Conclusions: All the patients reviewed presented some symptoms and signs pointing to brain stem involvement. Only $8 \%(2 / 24)$ had an acute course with no adequate warning signs.
\end{abstract}

initial phase of the disease in patients who died or became severely disabled, we have analysed the early clinical features and patterns of evolution in 24 patients with proven severe symptomatic basilar artery occlusion.

\section{METHODS}

In order to devise a diagnostic strategy as well as efficient management guidelines in patients with basilar artery occlusion, the symptoms (what brought the patient to the physician's attention?), signs (what were the signs present initially on physical examination?), and pattern of evolution of the disease need to be known. In an attempt to document these points further, we carried out a retrospective review of the records of all patients admitted to our population based primary care centre over a five year period in whom the diagnosis of basilar artery occlusion-that is, complete blockage of the basilar arterial lumen with or without vertebral artery involvement-was confirmed by either conventional transfemoral angiography, magnetic resonance angiography (MRA), or necropsy. The decision to include patients who underwent MRA to confirm the vascular lesion is supported by several reports on magnetic resonance imaging (MRI) of vertebrobasilar vascular disease, as well as more recent studies using MRA which have shown that these techniques are reliable for the diagnosis of basilar artery occlusion (macrovascular disease). ${ }^{24-30}$ In addition to MRA, one of these latter seven patients also had conventional angiography with good anatomical correlation between these two diagnostic modes.

All neurological manifestations occurring before admission and the formal clinical evaluation were considered initial or warning symptoms. The neurological signs on admission were the objective physical findings present when the patient was knowledge of the natural history of the disease is now of paramount importance. Given the lack of reports about the 
first evaluated following the above complaints. In all cases, history taking and clinical examination was done by a neurologist. The final neurological picture consisted either of deep coma with quadriplegia, or of the locked-in syndrome (normal state of consciousness with complete motor deficit except for some eye movements). ${ }^{31}$ Only five patients had a less severe deficit.

To establish the temporal pattern leading to the final neurological event, we reviewed and analysed all initial symptoms in relation to each other and to their chronological occurrence. The "prodromal phase" was defined as a series of transient and spontaneously regressive symptoms or deficits, separated in time by symptom-free periods during which the patient had no complaints. The "progressive phase" started when new symptoms or deficits became permanent and led to one another in an additive fashion.

\section{RESULTS}

Twenty four patients (16 men, eight women) aged between 22 and 80 years (mean (SD), 60.3 (16.3) years) satisfied the inclusion criteria. Table 1 summarises the clinical findings and relevant diagnostic studies. Twelve patients evolved towards deep coma or stupor and seven towards the lockedin syndrome, the main distinction between these being the normal state of consciousness present in the latter; five patients had a less dramatic course but nevertheless with severe neurological deficits. Fourteen patients subsequently died, and all had a general and central nervous system necropsy; these patients included 12 with thrombosis secondary to severe atherosclerosis, one with embolism related to known atrial fibrillation, and one with possible vertebrobasilar dissection. For the remaining 10 patients, the localisation of the arterial occlusion was based on the angiographic findings (conventional angiography or MRA). The extent of the arterial occlusions is shown diagrammatically in fig 1 . As more than half the representations in fig 1 are based on necropsy data, emphasis was on occlusion of the basilar artery itself, regardless of the patency of other vessels or the degree of collateral flow.

\section{Initial manifestations}

Table 2 lists the major initial symptoms. In our series, motor weakness was the most common complaint and affected the entire right or left side of the body 10 times, one or two extremities five times, and one patient was quadriplegic from the onset. Three patients also had a combined unilateral face involvement. Speech difficulties consisted of dysarthria in 12 patients, mutism in two, and possible aphasia in one. Headaches were reported six times in the occipital or neck region, twice in the frontal region, and twice diffusely. Eight patients rapidly developed some degree of altered state of consciousness, mostly as exaggerated somnolence; three also had one or more episodes of transient loss of consciousness occurring, respectively, 14 days, 3 days, and 12 hours before the final neurological event. Visual disturbances were reported as diplopia or blurred vision in six patients and as positive/negative visual phenomena (light flashes or brown spots) in two. Mental changes were usually reported by the patient's relatives using descriptive terms such as "strange," "disoriented," or "confused." Sensory deficits were rarely reported and, when present, mainly described as paraesthesiae involving both hands or feet. Other miscellaneous symptoms not mentioned in table 2 included "malaise," described as a sensation of impending death or general uneasiness in 10 patients; breathing difficulties in four patients; abnormal movements referred to as "seizures" in five patients; and sudden hearing loss or tinnitus in two patients.

\section{Neurological features on admission}

Table 3 lists the objective findings present when the patients were first seen by a neurologist on admission to the hospital up to a maximum of 10 days before the final neurological event. Motor deficits were again the most prominent finding, varying from isolated monoparesis to full quadriplegia. In seven patients, a bilateral extensor plantar response (Babinski sign) could be elicited in the presence of only unilateral motor weakness. When present, ataxia was contralateral to the motor deficit on four occasions. Although mentioned only by three patients, facial palsy could be demonstrated in the majority of patients on initial clinical examination. In four patients it was of unilateral peripheral type; in five it was of unilateral central type; one had a right central and left peripheral facial palsy; and in nine no details of whether it was of central or peripheral type were given (three were bilateral). One patient had an isolated facial palsy without further motor involvement of the upper or lower extremities (although weakness of the left hand was mentioned, this could never be documented on clinical examination). Extrinsic or intrinsic oculomotor disturbances were present initially in 15 patients. Pupillary abnormalities including miosis were found in six patients (bilaterally in five) and unilateral mydriasis in one. The lower cranial nerves were involved as follows: glossopharyngeal nerve (IX) in five patients (four bilaterally), vagus nerve $(X)$ in four (one bilaterally), spinal accessory nerve (XI) in three (all unilateral), and hypoglossal nerve (XII) in 13 (four bilaterally). In addition, dysarthria was present in seven patients, anarthria in four, dysphonia in two, aphonia in one, and dysphagia in five. Hemihypaesthesia was crossed in only two of the five patients in whom sensory deficits were found. When present early, abnormal breathing patterns were described as periodic in two patients, apneustic in two, ataxic in two, and as central hyperventilation in one. On admission, none of the 24 patients was deeply comatose, but 11 were described as drowsy and one was stuporose.

\section{Time course}

In general, two main patterns of evolution could be identified.

The first presented as an acute onset without any warning symptoms, the final stage of the disease being reached in less than 10 minutes. This pattern was found in only two patients (one 33 year old man and one 36 year old woman), who both survived, although severely disabled (locked-in syndrome).

The second had a more progressive onset and could be further subdivided on the basis of the presence of warning symptoms ("chronic") or their absence ("subacute").

The "chronic" pattern was found in 13 patients with several episodes of transient neurological symptoms or deficits, each lasting a few minutes to less than six hours. The total duration of this warning phase ranged from 12 hours to two months. Six of these 13 patients also had a preceding neurological event up to two years before their brain stem stroke (four suggestive of vertebrobasilar transient ischaemic attacks and two involving the anterior circulation; all lasted 24 hours or less and left no deficit).

The "subacute" pattern was seen in nine patients and consisted of a slowly progressive course with worsening and additive neurological deficits. In further reviewing our patients, it also became evident that this represented a final common pathway, as all patients in the chronic group ultimately also had a subacute phase leading to the final neurological event.

Once permanent and non-remitting neurological deficits were present, evolution to the final neurological picture was complete within six hours in nine cases, between six and 24 hours in seven cases, and in two to three days in six cases. 
Table 1 Summary of the clinical findings and relevant diagnostic studies for each patient

\begin{tabular}{|c|c|c|c|c|c|c|c|}
\hline $\begin{array}{l}\text { Unit } \\
\text { number }\end{array}$ & $\begin{array}{l}\text { Sex/age } \\
\text { (years) }\end{array}$ & $\begin{array}{l}\text { Initial } \\
\text { complaints (symptoms) }\end{array}$ & $\begin{array}{l}\text { Initial } \\
\text { neurological picture }\end{array}$ & $\begin{array}{l}\text { Final } \\
\text { neurological picture }\end{array}$ & MRA & $\begin{array}{l}\text { Conventional } \\
\text { angiography }\end{array}$ & Deceased \\
\hline 49742 & $M / 58$ & $\begin{array}{l}\text { Visual complaints, } \\
\text { mental changes, "seizure", } \\
\text { "malaise", altered } \\
\text { consciousness, R body } \\
\text { weakness, vertigo, speech } \\
\text { difficulties }\end{array}$ & $\begin{array}{l}\text { R head deviation, } R \text { miosis, } \\
\text { L VII paresis, bilat VIII } \\
\text { deficit, L X paresis, } \\
\text { R Babinski, R LE } \\
\text { hemiparesis, sleepiness }\end{array}$ & $\begin{array}{l}\text { Deep coma with flaccid } \\
\text { quadriplegia and periodic } \\
\text { respiration }\end{array}$ & ND & ND & Yes \\
\hline 146976 & $M / 33$ & Occipital headache & $\begin{array}{l}\text { See final neurological } \\
\text { Presentation }\end{array}$ & $\begin{array}{l}\text { Locked-in syndrome with } \\
\text { spastic quadriplegia and } \\
\text { eye movements only } \\
\text { maintained vertically }\end{array}$ & ND & $\begin{array}{l}\text { Occlusion of the } \\
\text { upper two thirds } \\
\text { of the basilar artery }\end{array}$ & $\begin{array}{l}\text { No } \\
y\end{array}$ \\
\hline 219272 & $M / 59$ & $\begin{array}{l}\text { Vertigo, frontal headache, } \\
\text { vomiting, altered } \\
\text { consciousness, speech } \\
\text { difficulties }\end{array}$ & $\begin{array}{l}\text { Bilat miosis, eye movement } \\
\text { abnormalities, nystagmus, } \\
\text { bilat VII palsy, bilat } \\
\text { Babinski, L UE plegia } \\
\text { and bilat LE paresis, } \\
\text { sleepiness }\end{array}$ & $\begin{array}{l}\text { Coma with flaccid } \\
\text { quadriplegia, ataxic } \\
\text { respiration, and } \\
\text { tachycardia }\end{array}$ & ND & ND & Yes \\
\hline 258466 & $M / 59$ & $\begin{array}{l}\text { Vomiting, altered } \\
\text { consciousness, speech } \\
\text { difficulties, R body } \\
\text { weakness, abnormal LE } \\
\text { movements }\end{array}$ & $\begin{array}{l}\text { R VII palsy, R Babinski, } \\
\text { R spastic hemiparesis, R LE } \\
\text { myoclonus, L cerebellar } \\
\text { ataxia, R hypaesthesia, } \\
\text { sleepiness }\end{array}$ & $\begin{array}{l}\text { Coma with eyes deviated } \\
\text { to the } \mathrm{R} \text {, quadriplegia and } \\
\text { extensor posturing }\end{array}$ & ND & $\begin{array}{l}\text { Occlusion of the } \\
\text { basilar artery and } \\
\mathrm{L} \text { vertebral artery }\end{array}$ & Yes \\
\hline 265666 & $\mathrm{~F} / 52$ & $\begin{array}{l}\text { L and occipital headache, } \\
\text { intermittent diplopia, } \\
\text { unsteady gait, vertigo, } \\
\text { hand and feet paraesthesiae, } \\
\text { vomiting, "seizure" }\end{array}$ & $\begin{array}{l}\text { Horizontal nystagmus, } \\
\text { slight L LE paresis }\end{array}$ & $\begin{array}{l}\text { Coma with spastic } \\
\text { quadriplegia, extensor } \\
\text { posturing, and apneustic } \\
\text { respiration }\end{array}$ & ND & ND & Yes \\
\hline 291011 & $\mathrm{~F} / 80$ & $\begin{array}{l}\text { "Malaise", vertigo, } \\
\text { speech difficulties, L body } \\
\text { weakness }\end{array}$ & $\begin{array}{l}\text { L VII palsy, L XII paresis, } \\
\text { dysarthria, bilat Babinski, } \\
\text { L hemiparesis, stupor }\end{array}$ & $\begin{array}{l}\text { Locked-in syndrome with } \\
\text { flaccid quadriplegia and } \\
\text { eye movements maintained } \\
\text { only for abduction of } R \text { eye }\end{array}$ & ND & ND & Yes \\
\hline 301435 & $M / 56$ & $\begin{array}{l}\text { Occipital headache, } \\
\text { "malaise", mental } \\
\text { changes, vertigo, } \\
\text { vomiting, L body weakness }\end{array}$ & $\begin{array}{l}\text { L VII palsy, bilat IX paresis, } \\
\text { L XII paresis, L Babinski, } \\
\text { L spastic hemiplegia }\end{array}$ & $\begin{array}{l}\text { Coma with spastic } \\
\text { quadriparesis, bilat. VII } \\
\text { palsy, and bilat. IX-XII } \\
\text { paresis }\end{array}$ & ND & $\begin{array}{l}\text { Distal } \mathrm{L} \text { vertebral } \\
\text { artery occlusion } \\
\text { and basilar } \\
\text { artery occlusion }\end{array}$ & No \\
\hline 304046 & $F / 33$ & $\begin{array}{l}\text { R UE weakness, frontal } \\
\text { headache, nausea and } \\
\text { vomiting, blurred vision, } \\
\text { "malaise", vertigo, } \\
\text { L facial weakness, speech } \\
\text { difficulties, "seizure" }\end{array}$ & $\begin{array}{l}\text { L VII palsy, L XII paresis, } \\
\text { R Babinski, R UE paresis, } \\
\text { transient respiratory } \\
\text { disturbances, sleepiness }\end{array}$ & $\begin{array}{l}\text { Locked-in syndrome with } \\
\text { flaccid quadriplegia, eye } \\
\text { movements only } \\
\text { maintained vertically, } \\
\text { extensor posturing, and } \\
\text { irregular respiration }\end{array}$ & ND & ND & Yes \\
\hline 318822 & $M / 61$ & $\begin{array}{l}\text { Bilat LE weakness, } \\
\mathrm{R} \text { retro-auricular and } \\
\text { occipital headache, } \\
\text { hearing loss, speech } \\
\text { difficulties }\end{array}$ & $\begin{array}{l}\text { Eye movement } \\
\text { abnormalities, bilat VII } \\
\text { palsy, bilat XII } \\
\text { paresis, bilat Babinski, } \\
\text { quadriplegia, irregular } \\
\text { respiration, hypersudation }\end{array}$ & $\begin{array}{l}\text { Locked-in syndrome with } \\
\text { spastic quadriplegia, } \\
\text { eye movements only } \\
\text { maintained vertically, and } \\
\text { hyperventilation }\end{array}$ & ND & ND & Yes \\
\hline 407078 & $F / 76$ & $\begin{array}{l}\text { Mental changes, speech } \\
\text { difficulties, unsteady gait, } \\
\text { diplopia }\end{array}$ & $\begin{array}{l}\text { Eye movement } \\
\text { abnormalities, horizontal } \\
\text { nystagmus, L VII palsy, } \\
\text { LXI paresis, bilat. } \\
\text { XII paresis, bilat Babinski, } \\
\text { R hemiparesis, sleepiness }\end{array}$ & $\begin{array}{l}\text { Coma with quadriplegia } \\
\text { and extensor posturing }\end{array}$ & ND & ND & Yes \\
\hline 444614 & $M / 80$ & $\begin{array}{l}\text { Occipital headache, } \\
\text { unsteady gait, nausea, } \\
\text { altered consciousness, } \\
\text { speech difficulties, } \\
\text { bilat UE paraesthesiae }\end{array}$ & $\begin{array}{l}\text { Bilat miosis, eye movement } \\
\text { abnormalities, horizontal } \\
\text { nystagmus, R VII palsy, } \\
\text { R IX-XII paresis, } \\
\text { R hemiparesis, R cerebellar } \\
\text { ataxia, L hypaesthesia, } \\
\text { sleepiness }\end{array}$ & $\begin{array}{l}\text { Coma with flaccid } \\
\text { quadriplegia }\end{array}$ & ND & ND & Yes \\
\hline 454699 & $M / 69$ & $\begin{array}{l}\text { "Malaise", nausea, } \\
\text { headache, diplopia, } \\
\text { vomiting, altered } \\
\text { consciousness, abnormal } \\
\text { movements, respiratory } \\
\text { disturbances, speech } \\
\text { difficulties }\end{array}$ & $\begin{array}{l}\text { Eye movement } \\
\text { abnormalities, } \\
\text { L XII paresis, L Babinski, } \\
\text { L hemiplegia, periodic } \\
\text { breathing pattern, } \\
\text { sleepiness }\end{array}$ & $\begin{array}{l}\text { Coma with spastic } \\
\text { quadriplegia, extensor } \\
\text { posturing, and ataxic } \\
\text { breathing pattern }\end{array}$ & $\begin{array}{l}\text { Absent flow- } \\
\text { void in the } \\
\text { basilar } \\
\text { artery }\end{array}$ & ND & Yes \\
\hline 517226 & $M / 72$ & $\begin{array}{l}\text { Vertigo, tinnitus, R UE } \\
\text { weakness, altered } \\
\text { consciousness, respiratory } \\
\text { disturbances }\end{array}$ & $\begin{array}{l}\mathrm{R} \text { VII palsy, } \mathrm{R} \mathrm{X} \text { and XII } \\
\text { paresis, bilat Babinski, } \\
\mathrm{R} \text { hemiplegia and L LE } \\
\text { paresis }\end{array}$ & $\begin{array}{l}\text { Coma with spastic } \\
\text { quadriplegia and } \\
\text { complete ophthalmoplegia }\end{array}$ & ND & ND & Yes \\
\hline 546809 & $M / 22$ & $\begin{array}{l}\text { Mental changes, altered } \\
\text { consciousness, unsteady } \\
\text { gait, R body weakness }\end{array}$ & $\begin{array}{l}\text { L mydriasis, R VII palsy, } \\
\text { horizontal nystagmus, } \\
\text { R Babinski, R flaccid } \\
\text { hemiparesis, sleepiness }\end{array}$ & $\begin{array}{l}\text { L mydriasis, } R \text { VII palsy, } \\
\text { bilat paresis } R>>L\end{array}$ & ND & $\begin{array}{l}\text { Occlusion of the } \\
\text { upper two thirds } \\
\text { of the basilar } \\
\text { artery }\end{array}$ & No \\
\hline 554272 & $\mathrm{~F} / 58$ & $\begin{array}{l}\text { "Malaise", vertigo, } \\
\text { vomiting, diplopia, L hand } \\
\text { paraesthesiae, L body } \\
\text { weakness, speech } \\
\text { difficulties }\end{array}$ & $\begin{array}{l}\text { Nystagmus, L VII palsy, } \\
\text { L Babinski, L spastic } \\
\text { hemiparesis, bilat } \\
\text { cerebellar ataxia, L } \\
\text { hypaesthesia, sleepiness }\end{array}$ & $\begin{array}{l}\text { Locked-in syndrome with } \\
\text { flaccid quadriplegia, eye } \\
\text { movements only maintained } \\
\text { horizontally, and flexor } \\
\text { posturing }\end{array}$ & ND & $\begin{array}{l}\text { Occlusion of the } \\
\text { basilar artery }\end{array}$ & Yes \\
\hline
\end{tabular}




\begin{tabular}{|c|c|c|c|c|c|c|c|}
\hline $\begin{array}{l}\text { Unit } \\
\text { number }\end{array}$ & $\begin{array}{l}\text { Sex/age } \\
\text { (years) }\end{array}$ & $\begin{array}{l}\text { Initial } \\
\text { complaints (symptoms) }\end{array}$ & $\begin{array}{l}\text { Initial } \\
\text { neurological picture }\end{array}$ & $\begin{array}{l}\text { Final } \\
\text { neurological picture }\end{array}$ & MRA & $\begin{array}{l}\text { Conventional } \\
\text { angiography }\end{array}$ & Deceased \\
\hline 622301 & $M / 75$ & $\begin{array}{l}\text { "Malaise", nausea, } \\
\text { vomiting, R UE } \\
\text { weakness, speech } \\
\text { difficulties }\end{array}$ & $\begin{array}{l}\text { Bilat miosis, vertical } \\
\text { nystagmus, eye movement } \\
\text { abnormalities, R facial } \\
\text { hypaesthesia, bilat VII } \\
\text { palsy, bilat VIII deficit, } \\
\text { bilat IX and R XII paresis, } \\
\text { R Babinski, R hemiparesis, } \\
\text { L hypaesthesia, sleepiness }\end{array}$ & $\begin{array}{l}\text { Coma with flaccid } \\
\text { quadriplegia, extensor } \\
\text { posturing, and periodic } \\
\text { respiration }\end{array}$ & ND & ND & Yes \\
\hline 634771 & $F / 36$ & $\begin{array}{l}\text { (Abnormal breathing, } \\
\text { no UE or LE movements) }\end{array}$ & $\begin{array}{l}\text { See final neurological } \\
\text { picture }\end{array}$ & $\begin{array}{l}\text { Locked-in syndrome with } \\
\text { spastic quadriplegia and } \\
\text { eye movements only } \\
\text { maintained vertically } \\
\text { down }\end{array}$ & ND & $\begin{array}{l}\text { Occlusion of the } \\
\text { basilar artery }\end{array}$ & No \\
\hline 668472 & $M / 76$ & $\begin{array}{l}\text { L hand weakness, L facial } \\
\text { weakness, unsteady gait }\end{array}$ & $\begin{array}{l}\text { L VII palsy, cerebellar } \\
\text { ataxia }\end{array}$ & $\begin{array}{l}\text { Horizontal nystagmus, } \\
\text { L VII palsy, R VIII deficit, } \\
\text { R hyperreflexia with } \\
\text { ankle clonus, R LE paresis, } \\
\text { L cerebellar ataxia }\end{array}$ & $\begin{array}{l}\text { Absent } \\
\text { flow-void } \\
\text { in the basilar } \\
\text { artery and } \\
L \text { vertebral } \\
\text { artery }\end{array}$ & ND & No \\
\hline 686644 & $M / 75$ & $\begin{array}{l}\text { "Malaise", altered } \\
\text { consciousness, L body } \\
\text { weakness }\end{array}$ & $\begin{array}{l}\text { Horizontal nystagmus, } \\
\text { L facial hypaesthesia, } \\
\text { L VII palsy, bilat IX paresis, } \\
\text { L XI paresis, bilat XII paresis, } \\
\text { L Babinski, L flaccid } \\
\text { hemiparesis, L hypaesthesia, } \\
\text { sleepiness }\end{array}$ & Coma with quadriplegia & $\begin{array}{l}\text { Absent } \\
\text { flow-void in } \\
\text { the basilar } \\
\text { artery }\end{array}$ & ND & No \\
\hline 724519 & $\mathrm{~F} / 58$ & $\begin{array}{l}\text { Diffuse } L \text { headache, } \\
\text { vomiting, mental } \\
\text { changes, speech } \\
\text { difficulties, } \\
\text { L body weakness }\end{array}$ & $\begin{array}{l}\text { L VII palsy, L IX paresis, } \\
\text { L Babinski, L UE paresis }\end{array}$ & $\begin{array}{l}\text { Horizontal nystagmus, } \\
\text { L VII palsy, L IX and X } \\
\text { paresis, bilat. Babinski, } \\
\text { L hemiplegia and R UE } \\
\text { paresis }\end{array}$ & $\begin{array}{l}\text { Absent } \\
\text { flow-void } \\
\text { in the basilar } \\
\text { artery }\end{array}$ & ND & No \\
\hline 725641 & $M / 73$ & $\begin{array}{l}\text { Visual disturbances, } \\
\text { bilat UE paraesthesiae, } \\
\text { breathing difficulties }\end{array}$ & $\begin{array}{l}\text { See final neurological } \\
\text { picture }\end{array}$ & $\begin{array}{l}\text { Locked-in syndrome } \\
\text { with flaccid quadriplegia } \\
\text { and eye movements only } \\
\text { maintained vertically }\end{array}$ & ND & ND & Yes \\
\hline 758564 & M/75 & $\begin{array}{l}\text { "Malaise", visual } \\
\text { disturbances, unsteady } \\
\text { gait, speech difficulties }\end{array}$ & $\begin{array}{l}\text { Bilat miosis, R superior } \\
\text { facial hypaesthesia, } \\
\text { bitonal voice, R cerebellar } \\
\text { ataxia }\end{array}$ & $\begin{array}{l}\text { Horizontal nystagmus, } \\
\text { R Babinski, R cerebellar } \\
\text { ataxia, L hypaesthesia, } \\
\text { sleepiness }\end{array}$ & $\begin{array}{l}\text { Absent } \\
\text { flow-void in } \\
\text { the basilar } \\
\text { artery and } \\
\text { R vertebral } \\
\text { artery }\end{array}$ & ND & No \\
\hline 776713 & $\mathrm{~F} / 48$ & $\begin{array}{l}\text { "Malaise", speech } \\
\text { difficulties, R body } \\
\text { weakness }\end{array}$ & $\begin{array}{l}\text { R VII palsy, bilat Babinski, } \\
\text { R spastic hemiplegia, } \\
\text { abnormal R hand } \\
\text { movements }\end{array}$ & $\begin{array}{l}\text { Stupor, erratic eye } \\
\text { movements, R XII paresis, } \\
\text { bilat Babinski, } \\
\text { R hemiplegia }\end{array}$ & $\begin{array}{l}\text { Absent } \\
\text { flow-void in } \\
\text { the basilar } \\
\text { artery }\end{array}$ & $\begin{array}{l}\text { Occlusion of the } \\
\text { basilar artery }\end{array}$ & No \\
\hline 781041 & $M / 61$ & $\begin{array}{l}\text { Occipital headache, } \\
\text { unsteady gait, R UE } \\
\text { weakness and clumsiness, } \\
\text { speech difficulties, } \\
\text { R facial weakness }\end{array}$ & $\begin{array}{l}\text { R VII palsy, bilat Babinski, } \\
\text { Slight R UE paresis, } \\
\text { R cerebellar ataxia }\end{array}$ & $\begin{array}{l}\text { Horizontal nystagmus, } \\
\text { R VII palsy, bilat XII } \\
\text { paresis, bilat cerebellar } \\
\text { ataxia, R hemiparesis, } \\
\text { R hypaesthesia }\end{array}$ & $\begin{array}{l}\text { Absent } \\
\text { flow-void in } \\
\text { he lower two } \\
\text { thirds of the } \\
\text { basilar artery }\end{array}$ & ND & No \\
\hline
\end{tabular}

The initial complaints are listed in the order of occurrence and represent the symptoms described by the patient or their relatives. Under initial neurological picture are listed only the abnormal findings on admission.

Bilat, bilateral; L, leff; LE, lower extremity; MRA, magnetic resonance angiography; ND, not done; R, right; UE, upper extremity; VII, facial nerve; $I X$, glossopharyngeal nerve; $\mathrm{X}$, vagus nerve; $\mathrm{XI}$, spinal (accessory) nerve; XII, hypoglossal nerve.

Analysis of the initial symptoms showed that headaches were the earliest complaint, closely followed by visual disturbances. During progression towards the final event, episodes of vertigo, nausea, and vomiting become more frequent. Articulatory difficulties and motor deficits were the ultimate warning signs, usually occurring six to 72 hours before the final neurological picture.

\section{DISCUSSION}

There are only a few published reports outlining the early symptoms and signs of basilar artery occlusion. This aspect is frequently summarised and incorporated in a more general discussion of the disease. We report here on 24 patients representing a pure sample of cases of basilar artery occlusion, as the diagnosis was formally confirmed by angiography or necropsy. Although no pathognomonic presentation could be identified, vertigo is often reported as an early symptom, conceivably indicating vertebrobasilar pathology. ${ }^{32}$ Other non-specific but prominent complaints included headaches, nausea, and vomiting, the latter two mostly associated with vertigo (vertigo alone was found in only three of the 24 patients). Together they all seem to represent early warning signs. Motor symptoms with relative few sensory complaints are often reported as a hallmark of brain stem ischaemic events. They most often appear as articulatory speech difficulties (as opposed to aphasic syndromes in cortical infarcts), visual disturbances ("blurred vision" most probably representing subclinical diplopia), or frank motor deficits. They were late signs, heralding a major impending neurological event. These findings are in accordance with a review of the published reports in which early symptoms were mentioned (396 patients in all $^{33-40}$ ) (table 4). It is also noteworthy that roughly one third of our patients showed altered wakefulness, mental status changes, and ataxia as presenting symptoms. Furthermore five patients presented with what witnesses considered a "seizure," or at least some abnormal clonic, myoclonic, or other repetitive limb movements. Similar accounts of such movements can be 


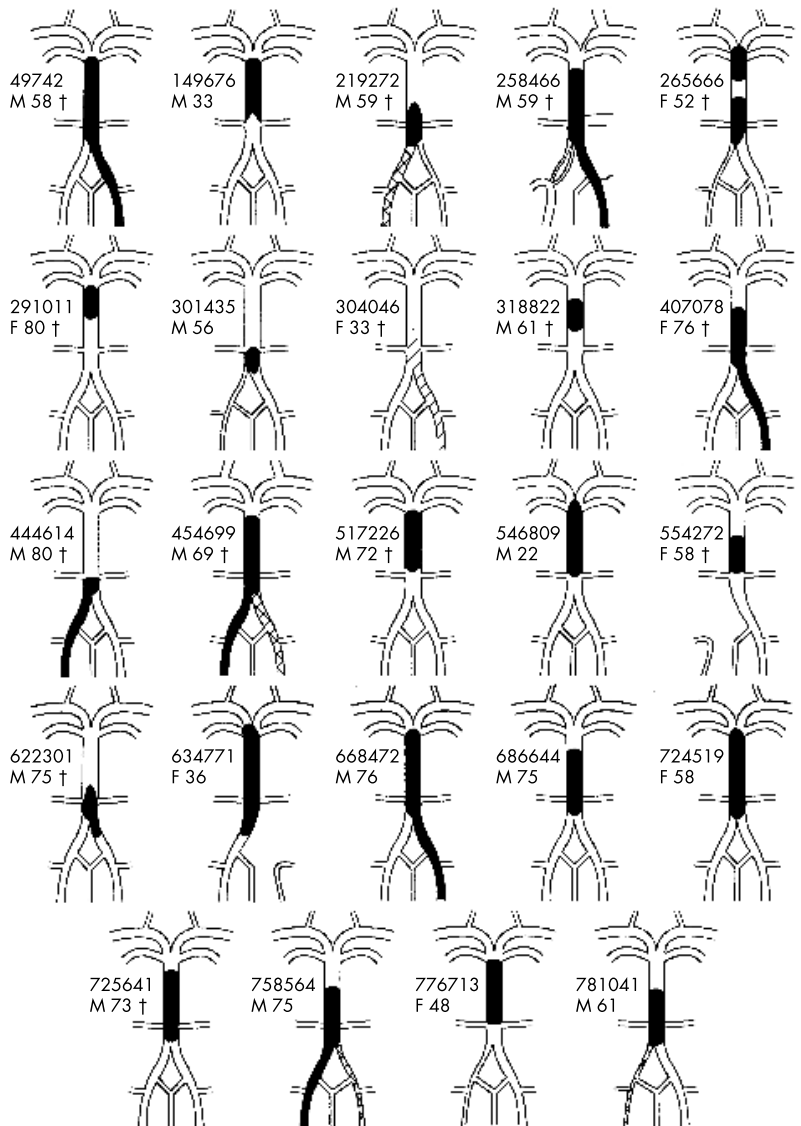

Figure 1 Schematic representation of the extent of basilar artery occlusion (black) and other abnormalities (stenosis (cross hatched) and dissection (hatched)) as determined by necropsy, conventional angiography, or magnetic resonance angiography. The patient's right side is on the left side of the drawing. For each patient, the unit number, sex, and age (in years) are given; tdenotes that the patient has died.

found elsewhere (totalling 1.5\% of cases). ${ }^{12519333436}$ These convulsive-like phenomena are often difficult to distinguish from real convulsions, but according to one investigator could help direct the diagnosis towards basilar artery occlusion. ${ }^{41}$ None of our patients had suffered from seizures before, and no cortical lesion was found at necropsy.

Clinical examination often reveals more deficits than could initially be inferred from the patient's complaints alone. Thus, although only three patients mentioned facial weakness, 19 had a facial palsy on initial examination. Characteristic eye movement abnormalities, lower cranial

Table 2 Initial complaints (symptoms) in 24 patients, in order of frequency

\begin{tabular}{ll}
\hline Symptom & $\mathbf{n}$ \\
\hline Motor deficits & 16 \\
Speech difficulties & 15 \\
Headaches & 10 \\
Nausea and/or vomiting & 10 \\
Vertigo & 8 \\
Visual disturbances & 8 \\
Altered consciousness & 8 \\
Unsteady gait & 7 \\
Mental changes & 5 \\
"Seizures" & 5 \\
Sensory disturbances & 4 \\
\hline
\end{tabular}

Table 3 Objective signs present on initial neurological examination

\begin{tabular}{ll} 
Sign & $\mathbf{n}$ \\
\hline Supranuclear or internuclear eye movement disturbances & \\
Internuclear ophthalmoplegia & 2 \\
"One-and-a-half" syndrome & 2 \\
Vertical gaze paresis & 2 \\
Horizontal gaze paresis & 4 \\
Ocular "bobbing" & 1 \\
"Skew deviation" & 2 \\
Nuclear or infranuclear eye movement disturbances & 2 \\
Nystagmus & 8 \\
Pupillary abnormalities & 7 \\
VII palsy & 19 \\
VIII deficit & 3 \\
IX-XII paresis & 15 \\
Bilateral extensor plantar response & 9 \\
Monoparesis & 5 \\
Hemiparesis or hemiplegia & 13 \\
Quadriplegia & 4 \\
Cerebellar ataxia & 5 \\
Hemihypaesthesia & 5 \\
Altered consciousness & 12 \\
Hypersudation & 2 \\
Abnormal breathing pattern & 7
\end{tabular}

Table 4 Comparison between the relative frequencies of different symptoms or symptom groups in our own series and in 396 patients compiled from published reports (references in the text)

\begin{tabular}{lll}
\hline & $\begin{array}{l}\text { This series } \\
(\mathbf{n = 2 4 )}\end{array}$ & $\begin{array}{l}\text { Other reports } \\
(\mathbf{n = 3 9 6 )}\end{array}$ \\
\hline Motor deficits including facial palsies & $67 \%$ & $42 \%$ \\
Speech difficulties & $63 \%$ & $30 \%$ \\
Vertigo, nausea, or vomiting & $54 \%$ & $73 \%$ \\
Headaches & $42 \%$ & $41 \%$ \\
Visual disturbances & $33 \%$ & $21 \%$ \\
Altered consciousness & $33 \%$ & $17 \%$ \\
Unsteady gait, ataxia & $29 \%$ & $6 \%$ \\
Mental changes & $21 \%$ & $5 \%$ \\
Sensory disturbances & $17 \%$ & $12 \%$
\end{tabular}

nerve deficits, crossed findings, bilateral extensor plantar responses, and prominent motor deficits with paucity of sensory findings are signs suggesting brain stem involvement. ${ }^{42} 43$ All our patients presented at least one of these findings, partially mimicking the final illness. Hypersudation and abnormal breathing patterns are usually late signs, reflecting involvement of the brain stem autonomic centres.

Recent experience with fibrinolysis has proved that the best results are achieved when this treatment is started before considerable brain stem function is lost. ${ }^{14}{ }^{44}$ We have shown that once neurological decline has begun, it takes at least six hours and up to three days to reach the final neurological picture. In more than half the patients, this decline was heralded by several warning episodes of waxing and waning neurological symptoms. Nearly all patients sought medical attention before the final illness had started. Awareness of this pattern of evolution could allow treatment to be directed more effectively and thus improve the outcome of basilar artery occlusion. We found only two patients (8\%) in whom occlusion of the basilar artery presented as an acute event from the outset, leaving no chance of salvage treatment. Both were young adults (in their early thirties), heavy smokers, and one was using oral contraceptives. In the absence of cardiac or haematological pathology, cerebral angiography showed vessel irregularities compatible with 
diffuse atherosclerosis. Although an embolic event could be postulated from the time course alone, not all embolic occlusions of the basilar artery had such an acute course.

The small size of our series did not permit statistically significant conclusions regarding the possible correlation between the symptoms and signs described or the various patterns of evolution and the anatomical level of the arterial occlusion. While it appears that patients with a proximal lesion tend to present with a more "chronic" course, this would need further verification in another study on a larger population of patients.

Several investigators have reported incidental angiographic findings of basilar artery occlusion in oligosymptomatic or asymptomatic patients. ${ }^{293645}$ These patients usually have a benign course and probably do not require any therapeutic intervention. Owing to our small sample size and the lack of a control group, the positive and negative predictive value of the reported symptoms and signs cannot be formally assessed. Pending further studies, comparison of the early symptoms and signs, together with the temporal profiles described in our study, with those found in this category of oligosymptomatic patients might allow the latter group to be distinguished from patients who will ultimately develop severe neurological deficits and who might have benefited from fibrinolysis.

\section{ACKNOWLEDGEMENTS}

We are grateful to Professor R Janzer from the Department of Neuropathology for access to the detailed necropsy data.

\section{Authors' affiliations}

G von Campe, F Regli, J Bogousslavsky, Department of Neurology,

Centre Hospitalier Universitaire Vaudois (CHUV), Lausanne, Switzerland

Competing interests: none declared

\section{REFERENCES}

1 Leyden E. Ueber die Thrombose der Basilar-Arterie. Z Klin Med 1882;5: 165-85

2 Kubik CS, Adams RD. Occlusion of the basilar artery - a clinical and pathological study. Brain 1946;69:73-121.

3 Biemond A. Thrombosis of the basilar artery and the vascularization of the brain stem. Brain 1951;74:300-17.

4 Haugsted H. Occlusion of the basilar artery. Diagnosis by vertebral angiography during life. Neurology 1956;6:823-8.

5 Freeman I, Ellis WRJ, Knox U. Occlusion of the basilar artery. An unusual case with recovery. Neurology 1953;3:154-6.

6 Cossa P, Boucebci M, Darcourt G. Un nouveau cas de thrombose complète du tronc basilaire avec récupération partielle et survie actuelle de près de deux ans. Rev Neurol (Paris) 1965;113:635-6.

7 Obrador S, Reinoso-Suarez F, Carbonell J, et al. Comatose state maintained during eight years following a vascular ponto-mesencephalic lesion. Electroencephalogr Clin Neurophysiol 1975;38:21-6.

8 Al-Wardi DAM, Adams AH, Hamilton AE. Four cases of "locked-in" syndrome and review of the literature. Bull Los Angeles Neurol Soc 1975;40:60-70

9 Caplan LR. Occlusion of the vertebral or basilar artery. Follow up analysis of some patients with benign outcome. Stroke 1979:10:277-82.

10 Labauge $R$, Pagès $M$, Blard JM. Survie prolongée après occlusion du tronc basilaire. 4 cas. Rev Neurol (Paris) 1989;145:789-94.

11 Zeumer H, Hacke W, Kolmann HL, et al. Lokale Fibrinolysetherapie bei Basilaris-Thrombose. Deutsch Med Wochenschr 1982;107: 728-31.

12 Zeumer $\mathrm{H}$. Vascular recanalizing techniques in interventional neuroradiology J Neurol 1985;231:287-94.

13 Hacke W, Zeumer H, Ferbert A, et al. Intra-arterial thrombolytic therapy improves outcome in patients with acute vertebrobasilar occlusive disease. Stroke 1988;19:1216-22.

14 Zeumer H, Freitag H-J, Grzyska U, et al. Local intraarterial fibrinolysis in acute vertebrobasilar occlusion. Technical developments and recent results. Neuroradiology 1989;31:336-40.
15 Tsakiris DA, Stock KW, Mürner J, et al. Lokale therapeutische Fibrinolyse bei ischämischen zerebrovaskulären Insulten: erste Ergebnisse bei 6 Patienten. Schweiz Med Wochenschr 1993;123:1784-9.

16 Henze T, Boeer A, Tebbe U, et al. Lysis basilar artery occlusion with tissue plasminogen activator. Lancet, 1987;ii:1391.

17 von Romatowski H-J, Henze T, Tebbe U. Rekanalisation eines Verschlusses $\operatorname{der}$ A. basilaris mit Gewebe-Plasminogen-Aktivator. Deutsch Med Wochenschr 1988;113:616.

18 Wildemann B, Hutschenreuter M, Krieger D. Infusion of recombinant tissue plasminogen activator for treatment of basilar artery occlusion. Stroke 1990;21:1513-14.

19 Herderscheê D, Limburg M, Hijdra A, et al. Recombinant tissue plasminogen activator in two patients with basilar artery occlusion. J Neurol Neurosurg Psychiatry 1991;54:71-3.

20 Kamps I, Weiss J, Ringelstein EB. Repetitive Behandlung einer subtotalen vertebrobasilären Stenose mit "tissue plasminogen activator" (†PA). Nervenarzt 1992;63:755-60.

21 Wijdicks EF, Nichols DA, Thielen KR, et al. Intra-arterial thrombolysis in acute basilar artery thromboembolism: the initial Mayo Clinic experience. Mayo Clin Proc 1997:72:1005-13.

22 Grond M, Rudolf J, Schmulling S, et al. Early intravenous thrombolysis with recombinant tissue-type plasminogen activator in vertebrobasilar ischemic stroke. Arch Neurol 1998:55:466-9.

23 Berg-Dammer E, Felber SR, Henkes $\mathrm{H}$, et al. Long-term outcome after local intra-arterial fibrinolysis of basilar artery thrombosis. Cerebrovasc Dis 2000;10:183-8.

24 Knepper L, Biller J, Adams HPJ, et al. MR imaging of basilar artery occlusion. J Comput Assist Tomogr 1990;14:32-5.

25 Schwaighofer BW, Klein MV, Lyden PD, et al. MR imaging of vertebrobasilar vascular disease. J Comput Assist Tomogr 1990;14:895-904.

26 Wentz KU, Röther J, Schwartz A, et al. MR-Angiographie des vertebrobasilären Stromgebiets: Einsatzmöglichkeiten der Saturationstechnik zur Bestimmung der Flussrichtung. Rofo Fortschr Geb Rontgenstr Neuen Bildgeb Verfahr 1992;156:120-4.

27 Maeder P, Meuli R, Gudinchet F, et al. Magnetic resonance angiography in vertebro-basilar ischemic accidents. Schweiz Rundsch Med Prax 1996;85:272-7.

28 Ruggieri PM, Masaryk TJ, Ross JS. Magnetic resonance angiography. Cerebrovascular applications. Stroke 1992;23:774-80.

29 Bogousslavsky J, Regli F, Maeder P, et al. The etiology of posterior circulation infarcts: a prospective study using magnetic resonance imaging and magnetic resonance angiography. Neurology 1993;43:1528-33.

30 Stock KW, Radue EW, Jacob AL, et al. Intracranial arteries: prospective blinded comparative study of MR angiography and DSA in 50 patients. Radiology 1995; 195:451-6.

31 Plum F, Posner JB. The diagnosis of stupor and coma. Philadelphia: FA Dvais Co, 1982:1-377.

32 Berlit P. Isolierter Schwindel bei vertebrobasilärer Ischämie - gibt es das? Hals-Nasen-Ohren-Heilunde Kopf und Hals-Chirurgie 1998:46:296-300.

33 Gauthier G. Contribution à l'étude de la thrombose basilaire (I). Schweiz Arch Neurol Neurochir Psychiatr 1962;90:209-34.

34 Gauthier G. Contribution à l'étude de la thrombose basilaire (II). Schweiz Arch Neurol Neurochir Psychiatr 1963:91:387-411.

35 Archer CR, Horenstein S. Basilar artery occlusion: clinical and radiological correlation. Stroke 1977;8:383-90

36 Labauge R, Pagès M, Marty-Double C, et al. Occlusion du tronc basilaire. Rev Neurol (Paris) 1981;137:545-71.

37 Liu J, Tuhrim S, Weinberger J, et al. Premonitory symptoms of stroke in evolution to the locked-in state. I Neurol Neurosurg Psychiatry 1983;46:221-6.

38 Patterson JR, Grabois M. Locked-in syndrome: a review of 139 cases. Stroke 1986; 17:758-64

39 Huber M, Hojer C, Schröder R. Klinische und pathologisch-anatomische Befunde bei akuten Basilaristhrombosen. Eine Untersuchung an 30 autoptisch gesicherten Fällen. Nervenarzt 1990;61:83-7.

40 Ferbert A, Brückmann HJ, Drummen R. Clinical features of proven basilar artery occlusion. Stroke 1990;21:1135-42.

41 Ropper AH. "Convulsions" in basilar artery occlusion. Neurology 1988:38:1500-1.

42 Siekert RG, Millikan CH. Studies in cerebrovascular disease. II. Some clinical aspects of thrombosis of the basilar artery. Proc Staff Meet Mayo Clin 1955;30:93-100.

43 Fisher CM. The "herald hemiparesis" of basilar artery occlusion. Arch Neurol 1988;45:1301-3

44 Willig V, Steiner T, Hacke W. Thrombolytische Therapie beim ischämischen Insult. Wien Klin Wochenschr 1997;109:795-803.

45 Meyer JS, Sheehan S, Baver RB. An arteriographic study of cerebrovascular disease in man. 1. Stenosis and occlusion of the vertebral-basilar arterial system. Arch Neurol 1960;2:27-45. 